\title{
Chlamydia trachomatis transmission between the oropharynx, urethra and anorectum in men who have sex with men: a mathematical model
}

\author{
Xianglong X ${ }^{1,2,3}$, Eric P. F. Chow ${ }^{2,3,4}$, Jason J. Ong ${ }^{1,2,3}$, Christian J. P. A. Hoebe ${ }^{5,6}$, Zhuoru Zou', Jane S. Hocking ${ }^{1,4}$,
} Christopher K. Fairley ${ }^{1,2,3}$ and Lei Zhang ${ }^{1,2,3,7^{*}}$ (D)

\begin{abstract}
Background: It has been presumed that Chlamydia trachomatis is transmitted between men only through anal or oral sex, but no mathematical models have tested this presumption.

Methods: To test this presumption, we created 20 compartmental mathematical models of different sexual practices that included both oral and anal sex and calibrated these models to the observed rates of Chlamydia trachomatis infection at three anatomical sites from 4888 men who have sex with men (MSM) in Melbourne Sexual Health Centre during 2018-2019.

Results: A model that included only oral and anal sex could replicate the observed rates of single-site infection at the oropharynx, urethra and rectum alone, but could not replicate infection at more than one of these sites (multisite). However, if we included transmission from sexual practices that followed one another in the same sexual episode (e.g. saliva contamination of the penis from oral sex transmitting chlamydia to the rectum by anal sex), we significantly improved the calibration of multisite infection rates substantially.
\end{abstract}

Conclusions: Our modelling study suggests that transmission routes other than just oral and anal sex are necessary to explain the high rate of Chlamydia trachomatis infection at more than one site.

Keywords: Transmission, Behavioural interventions, Sexual practices, Anatomical site, Chlamydia trachomatis, Men who have sex with men

\section{Background}

Chlamydia trachomatis (chlamydia) is a common sexually transmitted infection in men who have sex with men (MSM), and its burden is likely to rise over time as condom use falls in the pre-exposure prophylaxis (PrEP) era [1-4]. If the rates of condom use are to fall, new

\footnotetext{
* Correspondence: lei.zhang1@monash.edu

${ }^{1}$ China Australia Joint Research Center for Infectious Diseases, School of Public Health, Xi'an Jiaotong University Health Science Centre, Xi'an 710061, Shaanxi, People's Republic of China

${ }^{2}$ Melbourne Sexual Health Centre, Alfred Health, Melbourne, Australia Full list of author information is available at the end of the article
}

prevention measures will be needed, and these measures can only be developed if we clearly understand the transmission dynamics of chlamydia among MSM [5, 6].

Mathematical models are important for investigating the transmission of infections in populations, particularly when the transmission is complex or may be difficult to study epidemiologically $[7,8]$. The transmission of chlamydia may be more complex than has been previously appreciated given transmission occurs between three anatomical sites (oropharynx, urethra and anorectum), and there are a large number of potential sexual

(c) The Author(s). 2020 Open Access This article is licensed under a Creative Commons Attribution 4.0 International License which permits use, sharing, adaptation, distribution and reproduction in any medium or format, as long as you give appropriate credit to the original author(s) and the source, provide a link to the Creative Commons licence, and indicate if changes were made. The images or other third party material in this article are included in the article's Creative Commons licence, unless indicated otherwise in a credit line to the material. If material is not included in the article's Creative Commons licence and your intended use is not permitted by statutory regulation or exceeds the permitted use, you will need to obtain permission directly from the copyright holder. To view a copy of this licence, visit http://creativecommons.org/licenses/by/4.0/. The Creative Commons Public Domain Dedication waiver (http//creativecommons.org/publicdomain/zero/1.0/) applies to the data made available in this article, unless otherwise stated in a credit line to the data. 
practices that may transmit it. Indeed recent epidemiological studies have assessed some potential routes such as kissing, rimming (oral-anal sex) and the use of saliva as a lubricant for anal sex among MSM [9-11]. However, studying the potential contribution of these sexual practices to transmission using epidemiological studies is difficult because of the great number of questions that would be required for potentially many sexual partners. Such studies would also be difficult to analyse because men usually have multiple sexual practices in one sexual episode, making it hard to separate the individual contribution of each sexual practice. Several mathematical models have explored chlamydia transmission in heterosexuals [12-15], and also in MSM [16-20], but none of these studies used anatomical site-specific models. However, chlamydia can infect multiple anatomical sites, including the oropharynx, urethra and anorectum [10, 21-24]. Besides, the majority of MSM had multiple sexual practices in one sexual episode [25], which were not included in previous chlamydia models.
Given that little is known about chlamydia transmission, we progressively added other sexual practices to these two sexual practices to develop a series of mathematical models to determine what sexual practices were necessary to replicate the observed prevalence at each anatomical site (oropharynx, urethra and anorectum) in MSM.

\section{Methods}

After recovery from Chlamydia trachomatis infection, an individual can be immediately susceptible again [26]. We constructed a susceptible-infectioussusceptible compartmental model to test which transmission routes were necessary for chlamydia to replicate the observed infection rates at specific anatomical sites in MSM. Our Chlamydia trachomatis models are based on two previous anatomical sitespecific models [27, 28] (Additional file 1: Fig. S1).

\section{Model assumptions}

Our chlamydia models included the following assumptions related to multisite infections: (1) multisite

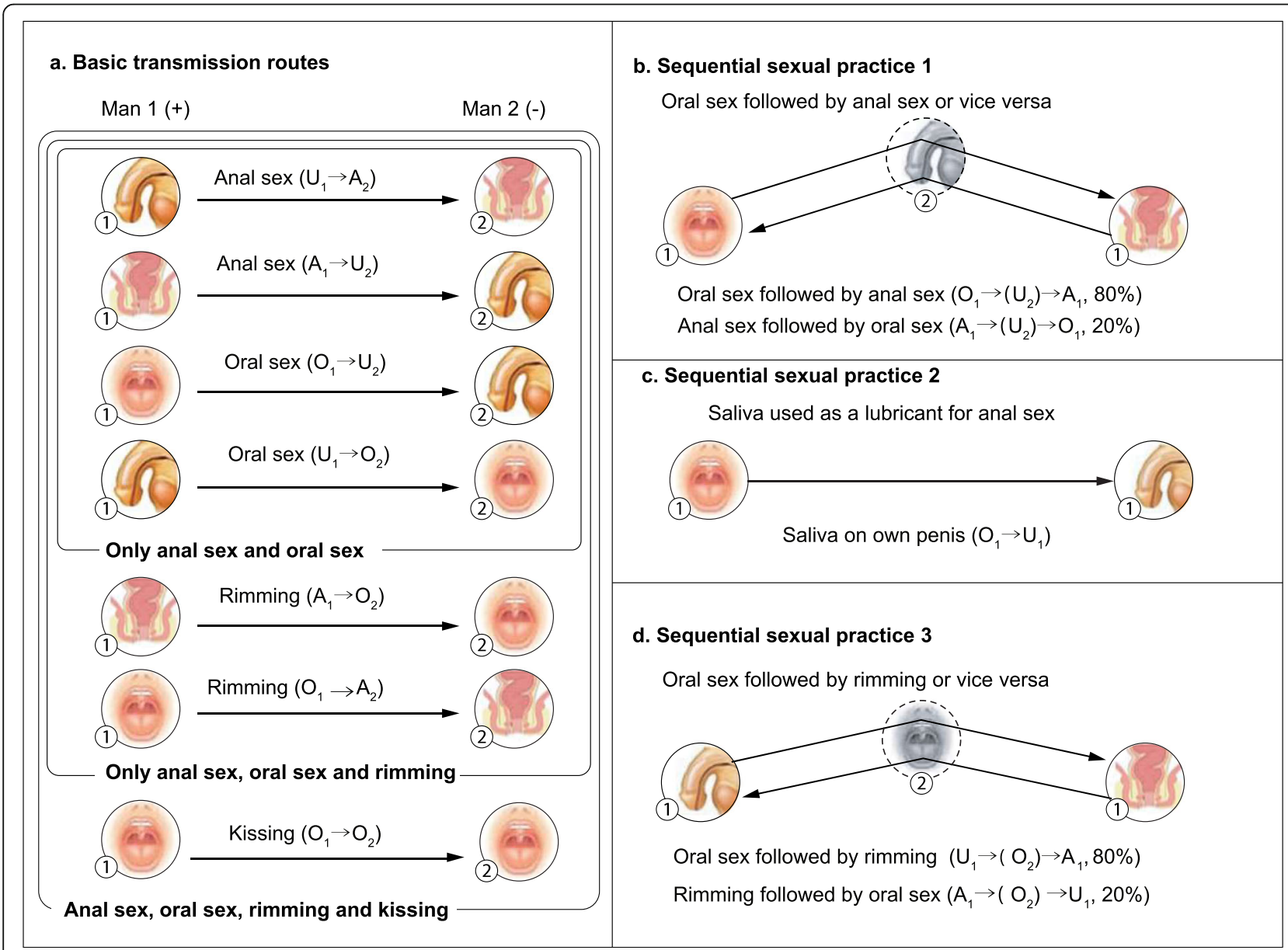

Fig. 1 Baseline transmission routes (a) to which transmission routes 1-3 (b-d) have been added; arrow signifies the direction of transmission. Note: Man 1 (1); Man 2 (2) 
infection could develop in a man who is already infected at one site when he has sex with another infected partner; and (2) within the same individual, and the Chlamydia trachomatis infection may be transmitted from one infected site to a different non-infected site through sexual practices during the same sexual episode ('sequential sexual practices') with another sex partner (outlined in Figs. 1 and 3) during a sequential sexual practice, we assumed that the medium anatomical site would act as a conduit for the transmission of the bacteria but not habour the bacteria at the site. As there currently is no evidence to inform the likelihood of infection at the medium anatomical site, we assumed that the medium anatomical site was not infected during a sequential sexual practice. This avoids adding substantial complexity to the model.

\section{Model development}

Given that little is known about chlamydia transmission other than from anal and oral sex, we systematically established 20 compartmental models to test the effect of adding in different sexual practices as outlined in Fig. 1. The transmission routes we tested included: (a) anal sex and oral sex only, (b) the addition of riming (oral-anal sex), (c) the addition of riming and kissing and (d) including the possibility that an individual with an infection at one site can be infected at another site (from the original site) during a sexual episode with another person whose sexual acts 'connect' these two anatomical sites in the same person. These 'connecting' sexual practices we have termed as 'sequential sexual practices'. The first sequential sexual practice was oral sex followed by anal sex (or the reverse) that allowed transmission from either the oral or anal site in the same person via chlamydia contamination on the partners' penis. For example, if an individual has oropharyngeal chlamydia and has receptive oral sex that was followed by anal sex, then remnant chlamydia containing saliva on the sexual partner's penis may transmit the infection from the mouth to the anus of the same individual via the partner's penis. The second sequential sexual practice was when saliva was used as a lubricant for anal sex and therefore may pass the infection from the oropharynx to an individual's own penis. The third sequential sexual practice was when either urethral or anal infection was passed to the urethra or anus (in the same person) via the partner's mouth when oral sex was followed by oral-anal sex (rimming) or anal-oral sex was followed by oral-penile sex. These sexual practices may be important for increasing the chance of an individual having the infection at more than one site (multisite infection), because they connect sites in the same man and multiple sexual practices in a single sexual encounter (e.g. oral sex followed by anal sex) is very common in MSM [25].
The models (model 1-20) were represented as a group of ordinary differential equations in Additional file 1 . We have not included other possible transmission routes into account. For example, we have assumed that transmission could not occur to the penis of one man from his partner's saliva during anal sex that follows oral sex (Fig. 1b).

\section{Model parameterization and calibration}

Our model parameters were from previously published biological and behavioural data of chlamydia (Additional file 2: Table S3) [2, 9, 20, 25, 27, 29-38] . We calibrated the models to diagnosis data of chlamydia infections at each anatomical site (i.e. oropharynx, urethra and anorectum) and multisite infection (oropharynx and urethra together, oropharynx and anorectum together, urethra and anorectum together, oropharynx and urethra and anorectum together) (Additional file 2: Table S4, 5).

We used six sources of data for model calibration. The first was data from the Melbourne Sexual Health Centre (MSHC) and included five similar studies on reported multisite infections of chlamydia. Urethral swabs, oropharyngeal swabs and anorectal swabs were tested for chlamydia by using nucleic acid amplification tests (NAAT) in MSHC [39]. To avoid the bias of multiple visits by the same returning patients, the model used data of chlamydia prevalence among 4888 MSM who visited MSHC for the first time and included 2565 MSM in 2018 and 2323 MSM in 2019 (Additional file 2: Table S4). The proportion of MSM who had more than one anatomical site infected with chlamydia was $20.0 \%$ (124/ 620). We also identified five similar studies reported multisite infections of chlamydia using NAAT as the validation data sets, including one study in Australia [40], two studies in the USA [41, 42], one study in the Netherlands [21] and one study in Thailand [22]. (Additional file 2: Table S5). We did not aim to collect all available multisite infections studies for chlamydia to cross-validate our findings.

The collected parameters of chlamydia models were sampled within the confidence interval by the Latin hypercube sampling method and repeated 300 times. By using the sum of squares of the errors to estimate the goodness of fit between simulation output and the epidemiological multisite infections data, we selected 'optimal runs' and we sorted the simulation outputs in descending order and selected the top 10\% simulations to generate the chlamydia models outputs with $95 \%$ confidence intervals. We measured the fitting error by calculating the sum of the squared errors. We compared chlamydia models using the minimal of sum squared errors between the empirical multisite infections data and the corresponding calibration results. We performed an 
independent $t$ test to check for differences in terms of the means of the sum of squared errors between two models. Statistical significance was considered at $p<$ 0.05 . The detailed calibration for models was provided in Additional file 1 [27, 36-38, 43, 44]. Output parameters for Chlamydia trachomatis models were provided in the additional file (Additional file 3: Tables S 8.1-8.219). We used MATLAB R 2019a to solve differential equations and conduct statistical analyses.

\section{Estimating the composition of chlamydia incidence}

We used our calibrated models to estimate the composition of chlamydia incidence caused by oropharyngeal, anorectal and urethral infection. Based on our previously reported method [27], we estimated the new infections at any given time and calculated the ratio between the number of new infections and the number of susceptible. We also used our models to estimate the proportion of incidence caused by sexual practices.

\section{Uncertainty and sensitivity analysis}

We conducted sensitivity analyses for the chlamydia models using the whole parameter set with varying some important parameters, including the duration of infection, frequency of sexual practices and the proportion of men with sequential sexual practices (details in Additional file 1).

\section{Results}

Replication of site-specific chlamydia prevalence without sequential sexual practices

The three models (models 1 to 3 ) that included no sequential sexual practices could only replicate single site infection at the oropharyngeal, urethra and anorectum, but could not replicate the high proportion of men with chlamydia at more than one site (multisite infection) (Additional file 4: Fig. S2a).

\section{Replication of site-specific chlamydia prevalence with sequential sexual practices}

We added the three sequential sexual practices that connected all three anatomical sites in the same person to model 1 (anal sex and oral sex only) to build models 46. Like models $1-3$, we were able to replicate single site infection at the oropharynx, urethra and anorectum in models 4-6. However, we were also able to improve the calibration of multisite infection substantially and significantly both at the oropharynx and anorectum when we added oral sex followed by anal sex (or vice versa) (model 4 vs. model $1(p<0.01)$ and model 6 vs. model 1 $(p<0.01))$. (Additional file 4: Fig. S8a; Additional file 2: Table S6).

We added the three sequential sexual practice that connected all three anatomical sites in the same person to model 2 (anal sex, oral sex and rimming) to build models 7-13. Like in models $4-6$, we were able to replicate single-site infection at the oropharyngeal, urethra and anorectum. Besides, we were also able to improve the calibration of multisite infection substantially and significantly both at the oropharynx and anorectum when we added oral sex followed by anal sex (or vice versa) to model 2 (models 7, 10, 11 and 13). Similarly, we could substantially improve the calibration of multisite infection at both the urethra and anorectum (Fig. 2a) when we added oral sex followed by rimming (or reverse) to model 2 (models 9, 11-13). According to the sum of squared errors, our best calibration model among all above 13 models (model 1-13) was model 11 (introduction of oral sex followed by anal sex (or vice versa) and oral sex followed by rimming (or vice versa)) (Additional file 2: Table S6).

We added the three sequential sexual practice that connects all three anatomical sites in the same person to model 3 (anal sex, oral sex, rimming and kissing only) to build models 14-20. Like in models 1-13, we were able to replicate single site infection at the oropharyngeal, urethra and anorectum in models 14-20. Furthermore, we were also able to improve the calibration of multisite infection substantially and significantly at both the oropharynx and anorectum when we added oral sex followed by anal sex (or vice versa). Similarly, we were able to substantially improve the calibration of multisite infection at both the urethra and anorectum when we added oral sex followed by rimming (or vice versa) (Additional file 4: Fig. S27a).

We compared the calibration of the models using the sum of squared errors and used the baseline model as model 1 (for models 4-6), model 2 (for model 7-13) and model 3 (for models 14-20). The calibration performance results of all 20 chlamydia models are reported in the additional file (Additional file 2: Table S6). We analysed the comparison of the means of the sum of squared error between model 11 and model 18, and the difference had no statistical significance $(p=0.0869)$.

\section{Estimate the relative proportions of incident chlamydia at different sites and due to different sexual practices}

We used model 2 and models $7-13$ to calculate the proportion of incident cases because these models provided the best calibration. The incidence of chlamydia infection varied by anatomic site (anorectal, range 50.963.7\%; urethral, range 31.4-43.6\%; and oropharyngeal, range $5.2-7.5 \%$ ), across all eight models (Fig. 3a). The proportion of incident chlamydia caused by anal sex only was 44.7-55.9\% (anorectum-to-urethra, range 14.0$30.8 \%$; urethra-to-anorectum, range $24.5-36.1 \%$ ), by riming only was $15.6-36.1 \%$ (anorectum-to-oropharynx, range $2.6-3.6 \%$; oropharynx to anorectum, $13.1-32.5 \%$ ), 


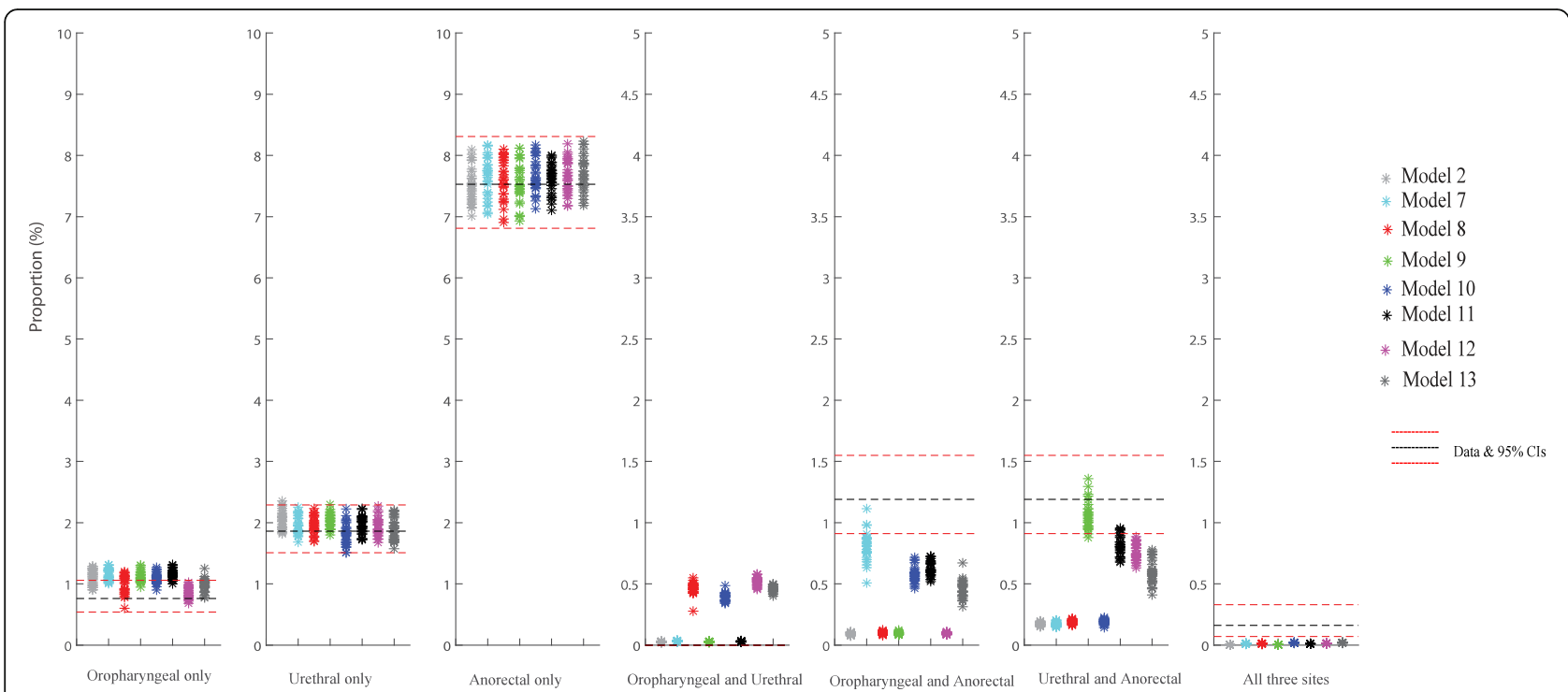

Fig. 2 Estimates of the eight models for the percentage of specific anatomical sites positive for Chlamydia trachomatis for the 8 models (model 2, 7-13) and the 95\% confidence intervals for the observed site-specific positivity among 4888 MSM attending Melbourne Sexual Health Centre in 2018 and 2019. The prevalence of oropharyngeal and urethral infection is zero and that therefore the dashed lines are missing. Model 2 (dark grey asterisk): anal and oral sex and rimming. Model 7 (cyan asterisk): anal sex and oral sex, rimming and sequential oral/anal sex. Model 8 (red asterisk): anal sex and oral sex and rimming and using saliva as a lubricant for anal sex. Model 9 (green asterisk): anal sex and oral sex and rimming and sequential oral sex/riming. Model 10 (blue asterisk): anal sex and oral sex and rimming, sequential oral/anal sex and using saliva as a lubricant for anal sex. Model 11 (black asterisk): anal sex and oral sex and rimming and sequential oral/anal sex and sequential oral sex/riming. Model 12 (magenta asterisk): anal sex and oral sex and rimming and using saliva as a lubricant for anal sex and sequential oral sex/riming. Model 13 (dim grey asterisk): anal sex and oral sex and rimming and sequential oral/anal sex and using saliva as a lubricant for anal sex and sequential oral sex/riming

by oral sex only was $8.7-19.2 \%$ (urethra-to-oropharynx, range 2.6-3.7\%; oropharynx-to-urethra, range 5.6$15.6 \%)$ and by all sequential activities was $7.3-23.6 \%$, across all eight models (Fig. 3b).

\section{Sensitivity analysis}

We performed sensitivity analyses of the models that provided the best calibration of multisite infection (models 2, 7-13). The results showed that varying key model indicators, including duration of infection, frequency of sexual practices and the proportion of men with sequential sexual practices, would not alter our conclusions relating to model calibration and incidence estimations. Including oral sex followed by anal sex (or reverse), saliva use as a lubricant for anal sex and oral sex followed by rimming (or reverse) were essential for calibrating multisite chlamydia infection. Anorectal infection and anal sex contributed the most to chlamydia incidence. We repeated the same analysis in all five validation datasets, and the results were essentially the same. Similarly, the five validation datasets showed that anorectal infections and anal sex contributed the most to chlamydia incidence (detailed results of sensitivity analysis in Additional file 4).

\section{Discussion}

This is the first modelling study to explore the role that different sexual practices play in chlamydia transmission at each anatomical site (oropharynx, urethra and anorectum) in MSM. We were unable to replicate the high proportion of chlamydia infection at more than one anatomical site without including some sequential sexual practices that transmit chlamydia between anatomical sites in the same person. Including oral sex followed by anal sex (or vice versa) improved the calibration of multisite infection at both the oropharynx and anorectum. Including oral sex followed by rimming (or vice versa) improved the calibration of multisite infection, both for the urethra and anorectum. Our optimal calibration results were obtained with oral sex, anal sex, riming and sequential oral and anal sex together with sequential oral sex and rimming. Our findings suggest that chlamydia transmission may be more complicated than had previously been appreciated, and therefore, the public health messages required to control it, in an environment with falling rates of condoms use may need to be more complex than just using condoms more. These hypothetical models of transmission and other potential ones that we did not explore will need to be confirmed in epidemiological studies, but our data will provide some guidance for the direction of these studies. 


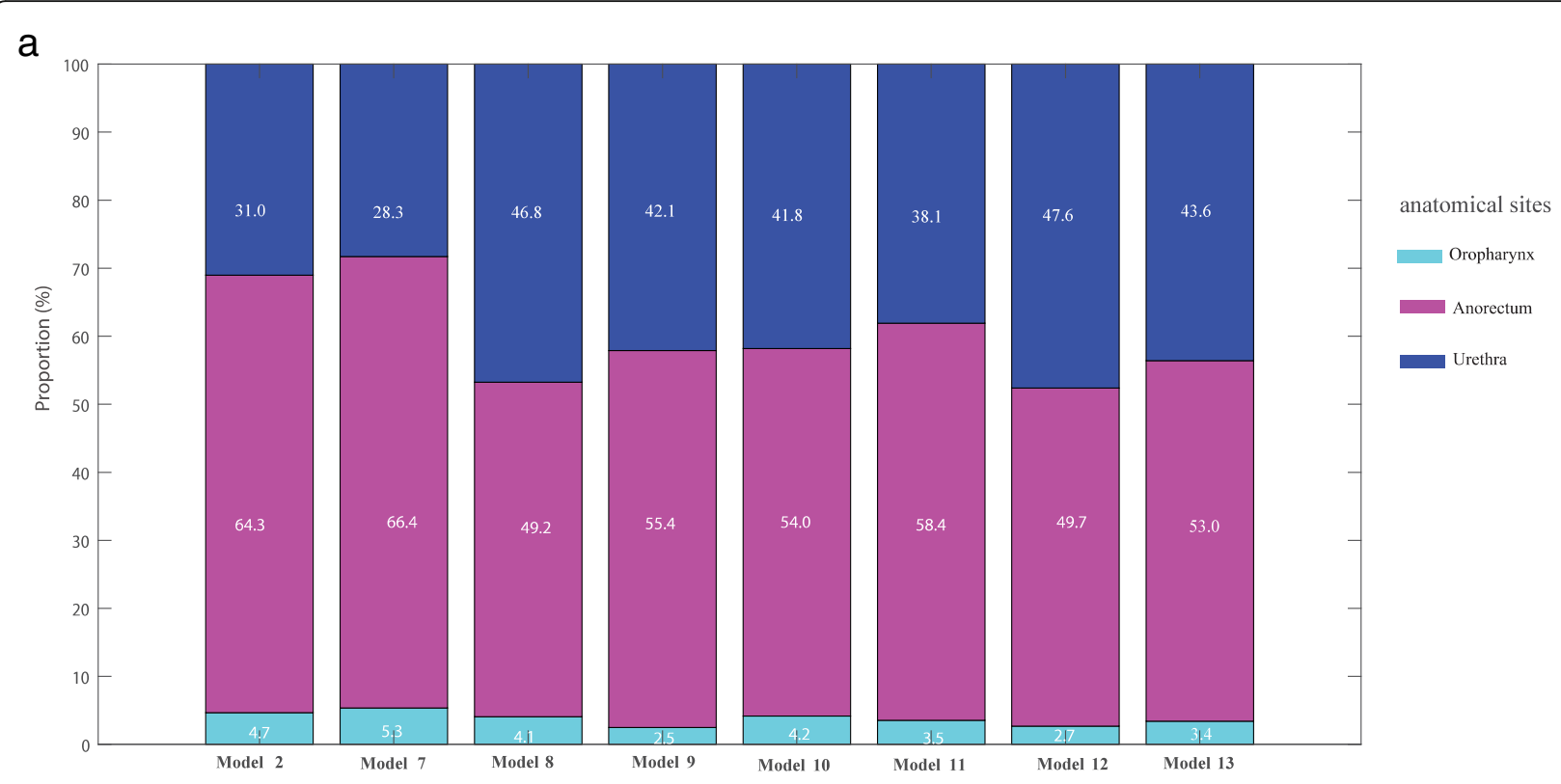

b

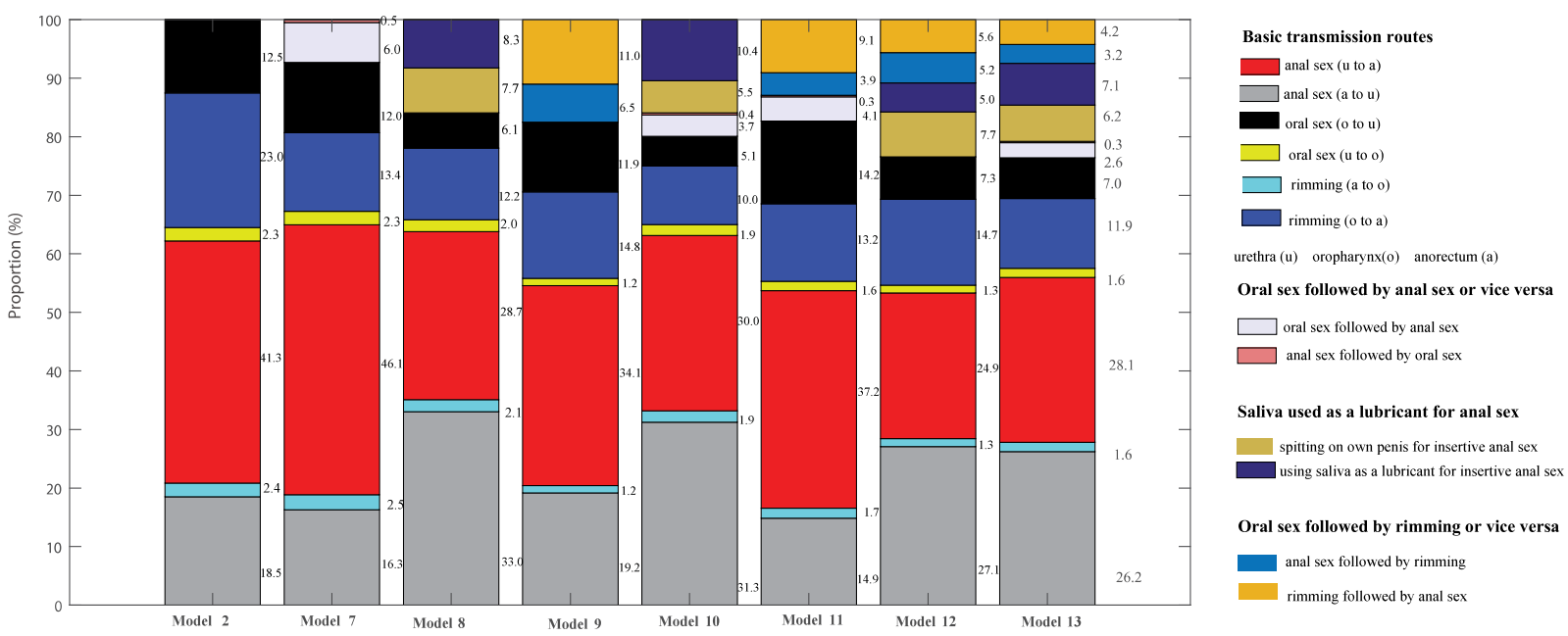

Fig. 3 a Estimated proportion of incident Chlamydia trachomatis cases that occur at the oropharynx, anorectum or urethra in MSM from the eight models (model 2, 7-13) among 4888 MSM attending Melbourne Sexual Health Centre in 2018 and 2019. Model 2: anal and oral sex and rimming. Model 7: anal sex and oral sex, rimming and sequential oral/anal sex. Model 8: anal sex and oral sex and rimming and using saliva as a lubricant for anal sex. Model 9: anal sex and oral sex and rimming and sequential oral sex/riming. Model 10: anal sex and oral sex and rimming, sequential oral/anal sex and using saliva as a lubricant for anal sex. Model 11: anal sex and oral sex and rimming and sequential oral/anal sex and sequential oral sex/riming. Model 12: anal sex and oral sex and rimming and using saliva as a lubricant for anal sex and sequential oral sex/ riming. Model 13: anal sex and oral sex and rimming and sequential oral/anal sex and using saliva as a lubricant for anal sex and sequential oral sex/riming and rimming and using saliva as a lubricant for anal sex. Model 9 (green asterisk): anal sex and oral sex and rimming and sequential oral sex/riming; Model 10 (blue asterisk): Anal sex and oral sex and rimming, sequential oral/anal sex and using saliva as a lubricant for anal sex. Model 11 (black asterisk): anal sex and oral sex and rimming and sequential oral/anal sex and sequential oral sex/riming. Model 12 (magenta asterisk): anal sex and oral sex and rimming and using saliva as a lubricant for anal sex and sequential oral sex/riming. Model 13 (dim grey asterisk): anal sex and oral sex and rimming and sequential oral/anal sex and using saliva as a lubricant for anal sex and sequential oral sex/ riming. $\mathbf{b}$ Estimated proportion of incident Chlamydia trachomatis cases caused by sexual practices in MSM from the eight models (model 2, 713) among 4888 MSM attending Melbourne Sexual Health Centre in 2018 and 2019. Model 2: anal and oral sex and rimming. Model 7: anal sex and oral sex, rimming and sequential oral/anal sex. Model 8: anal sex and oral sex and rimming and using saliva as a lubricant for anal sex. Model 9: anal sex and oral sex and rimming and sequential oral sex/riming. Model 10: anal sex and oral sex and rimming, sequential oral/anal sex and using saliva as a lubricant for anal sex; Model 11: Anal sex and oral sex and rimming and sequential oral/anal sex and sequential oral sex/riming. Model 12: anal sex and oral sex and rimming and using saliva as a lubricant for anal sex and sequential oral sex/riming. Model 13: anal sex and oral sex and rimming and sequential oral/anal sex and using saliva as a lubricant for anal sex and sequential oral sex/riming 
Our study has several limitations. First, we had to make a number of assumptions about the parameters when published data were not available or uncertainty. To address this issue, we performed sensitivity analyses for some of these variables. For example, we used different estimates of the duration of asymptomatic urethral and anal infection, frequency of sexual practices. Second, we did not include all possible sequential practices because little is known about them, and there are many possible permutations and combinations. Third, we also did not include transmission to the anatomical site that was acting as the conduit between two other sites during the sequential sexual practice. For example, we assumed that when oral sex is followed by anal sex that the penis could not be infected from the remnant saliva during anal sex. Similarly, we assumed that when anal sex is followed by oral sex that the penis could not be infected from remnant faecal material during oral sex. We acknowledge that this may happen but not only would it add substantial complexity to an already complicated model, but transmission between these sites could also occur through other acts (oral sex or anal sex). These transmission routes also do not address the key epidemiological issue that infection at more than one site in the same man is currently not well explained. We feel that our findings should be interpreted only as indicating that transmission is likely to be more complicated than had previously been appreciated, and transmission is unlikely to be solely from just anal and oral. Finally, our models did not include group sex because little is known about sequential sexual practices with more than one sex partner.

One important issue to consider is whether chlamydia can remain viable and infectious in genital secretions (e.g. saliva) and therefore remain infectious during sequential sexual acts. Chlamydial DNA is detected in genital secretions, including commonly in the saliva (69\%) of men who have oropharyngeal chlamydia [45]. Although there have not been studies to assess the viability of chlamydia in saliva, it is commonly reported to be viable in rectal samples (58\%) using mRNA and studies using culture suggest it may be viable in the environment [46, 47]. Researchers from The Netherlands particularly who have pioneered the viability assays suggest that inoculation from the vagina to the anus may be the cause for the high and unexplained rate of anal chlamydia in women [46]. Further studies will be required to clarify this important issue.

Our models suggested that oropharyngeal chlamydia incidence was relatively low in MSM and helped explain the epidemiological data showing that oropharyngeal chlamydia was not common [48, 49]. Our findings are consistent with chlamydia's higher affinity for columnar epithelium rather than the squamous epithelium that mostly covers the oropharynx [50]. It has been speculated that this is why there is a lower prevalence of chlamydia in the throat compared to Neisseria gonorrhoeae [40]. Our models indicated that anorectal infection significantly contributed to the overall incidence of chlamydia. Our model suggests that penile-anal sex is the main contributor to new chlamydia infections in MSM. Our findings suggest that consistent condom use for penile-anal sex could prevent more than half of incident cases and so promoting their use is an effective prevention strategy. Our estimated incidence data support the observed epidemiological data that anorectal chlamydia infection was the most common site-specific infection $[51,52]$. Our models suggested that the estimated proportion of incident chlamydia caused by all sequential activities while low (7.3-23.6\%) was necessary to replicate the observed epidemiology.

There are relatively few epidemiological studies investigating the transmission of chlamydia by sexual practices other than anal and oral sex in MSM. While it remains unclear, for example, whether sexual practices that involve saliva or saliva contamination could contribute to chlamydia transmission [10]. The Health In Men Study reported that insertive oral sex with ejaculation was associated with urethral chlamydia infection, and incident anal chlamydia was associated with receptive rimming [31]. A case-control study of chlamydial urethritis suggested that about $13 \%$ of cases were from oral sex or saliva exposure from oral sex [32]. However, a crosssectional study of MSM attending a sexual health service found that using saliva as a lubricant and rimming was not associated with the presence of chlamydia [10]. There have been only one study assessing kissing, and this study did not find that kissing was associated with oropharyngeal chlamydia [9]. These findings from epidemiological studies are generally consistent with our models.

Our study suggests that the transmission of chlamydia is more complex than had previously been appreciated. Our models found that inclusion of sequential sexual practices (oral sex followed by anal sex and oral sex followed by rimming) could improve the calibration of multisite infection substantially, particularly multisite infection of both the oropharynx and anorectum and multisite infection of both the urethra and anorectum. We thought it was important to assess more complex sexual practices because a previous study reported that most MSM had more than one sexual practice during the same sexual episode [25]. Our findings suggest that more complicated transmission routes may be required to explain multisite chlamydia prevalence, and, thus, chlamydia caused by sequential sexual practices deserve further attention. The findings of our study also could provide some direction for epidemiological research of 
the new transmission routes of chlamydia that our models suggest oral sex followed by anal sex (or reverse) and oral sex followed by rimming (or reverse) are necessary to replicate the epidemiological data.

\section{Conclusions}

Our study is the first modelling study to explore the role that different sexual practices play in chlamydia transmission at each anatomical site (oropharynx, urethra and anorectum) in MSM. Our modelling study suggests that transmission routes other than just oral and anal sex are necessary to explain the high rate of Chlamydia trachomatis infection at more than one site. Our modelling study may be useful to understand the transmission of Chlamydia trachomatis between the oropharynx, urethra and anorectum in men who have sex with men, thus making an essential step towards the control of chlamydia transmission at multiple anatomical sites in men.

\section{Supplementary information}

Supplementary information accompanies this paper at https://doi.org/10. 1186/s12916-020-01796-3.

\section{Additional file 1. Details of model equations, model calibration and sensitive analysis.}

Additional file 2: Table S1. Definition of Chlamydia trachomatis models based on sexual practices. Table S2. Sexual practices included in the 20 Chlamydia trachomatis models. Table S3. Biological and behavioural data of Chlamydia trachomatis for model parameterization in MSM. Table S4. The anatomical site-specific infection prevalence of Chlamydia trachomatis in MSM attending MSHC in 2018 and 2019. Table S5. All anatomical site-specific infection prevalence datasets of Chlamydia trachomatis for model calibration and validation. Table S6. Output parameters and transmission probability of the best-calibrated model and baseline model based on data from 4888 MSM attending Melbourne Sexual Health Centre. Table S7. Sum of squared error of 20 Chlamydia trachomatis models on data of 4888 MSM attending Melbourne Sexual Health Centre in 2018 and 2019.

Additional file 3. Output parameters for Chlamydia trachomatis models. Tables S 8.1-8.219.

Additional file 4. Additional results: model calibration, estimating the composition of incidence, and sensitivity analysis. Figs. S2a-32c.

\section{Abbreviations}

chlamydia: Chlamydia trachomatis; MSHC: Melbourne Sexual Health Centre; MSM: Men who have sex with men

\section{Acknowledgements}

We thank Afrizal at the MSHC for extracting the 2018-2019 MSHC data and Marjan Tabesh at the MSHC for providing the unpublished 2018-2019 MSHC data. We also thank Barbara Van Der Pol at the University of Alabama at Birmingham for providing data and insightful discussion. We thank Dr. van Liere GAFS for providing Dutch data. We thank Mark Chung at the MSHC for help in preparing Fig. 1.

\section{Authors' contributions}

$X L X, L Z$ and CKF conceived and designed the study. $X L X$ and $L Z$ established the models. XLX and LZ did the analysis. XLX wrote the first draft. CKF provided critical insights for framing of the first draft. EPFC, JJO, CJPAH, ZRZ and JSH revised the manuscript. All authors reviewed drafts and approved the final manuscript.

\section{Funding}

EPFC was supported by an Australian National Health and Medical Research Council (NHMRC) Emerging Leadership Investigator Grant (GNT1172873). CKF was supported by an Australian NHMRC Leadership Investigator Grant (GNT1172900). JJO was supported by an Australian NHMRC Early Career Fellowship (APP1104781). LZ was supported by the National Natural Science Foundation of China (Grant number: 8191101420), Outstanding Young Scholars Funding (Grant number: 3111500001), Xi'an Jiaotong University Basic Research and Profession Grant (Grant number: xtr022019003, xzy032020032) and Xi'an Jiaotong University Young Talent Support Grant (Grant number: YX6J004).

\section{Availability of data and materials}

All data analysed during this study are included in this article and its additional file.

\section{Ethics approval and consent to participate}

This study used secondary data analysis of datasets obtained from previous publications and therefore ethical approval was not required.

\section{Consent for publication}

Not applicable.

\section{Competing interests}

The authors declare that they have no competing interests.

\section{Author details}

${ }^{1}$ China Australia Joint Research Center for Infectious Diseases, School of Public Health, Xi'an Jiaotong University Health Science Centre, Xi'an 710061, Shaanxi, People's Republic of China. ${ }^{2}$ Melbourne Sexual Health Centre, Alfred Health, Melbourne, Australia. ${ }^{3}$ Central Clinical School, Faculty of Medicine, Nursing and Health Sciences, Monash University, Melbourne, Australia. ${ }^{4}$ Centre for Epidemiology and Biostatistics, Melbourne School of Population and Global Health, The University of Melbourne, Melbourne, Australia. ${ }^{5}$ Department of Sexual Health, Infectious Diseases and Environmental Health, South Limburg Public Health Service, Heerlen, The Netherlands. ${ }^{6}$ Department of Medical Microbiology, Care and Public Health Research Institute (CAPHRI), Maastricht University Medical Centre (MUMC+), Maastricht, The Netherlands. ${ }^{7}$ Department of Epidemiology and Biostatistics, College of Public Health, Zhengzhou University, Zhengzhou, Henan, People's Republic of China.

Received: 5 June 2020 Accepted: 24 September 2020 Published online: 17 November 2020

\section{References}

1. Thng CCM: A Review of Sexually Transmitted Infections in Australia Considerations in 2018. Acad Forensic Pathol. 2018;8(4):938-46.

2. HIV, viral hepatitis and sexually transmissible infections in Australia: annual surveillance report 2018. https://kirby.unsw.edu.au/report/hiv-viral-hepatitisand-sexually-transmissible-infections-australia-annual-surveillance.

3. Jane R, Melanie T. Chlamydia, gonorrhoea, trichomoniasis and syphilis: global and regional estimates of prevalence and incidence, 2016; 2019.

4. Chow EPF, Grulich AE, Fairley CK. Epidemiology and prevention of sexually transmitted infections in men who have sex with men at risk of HIV. Lancet HIV. 2019;6(6):e396-405.

5. Liu AY, Cohen SE, Vittinghoff E, Anderson PL, Doblecki-Lewis S, Bacon O, Chege W, Postle BS, Matheson T, Amico KR, et al. Preexposure prophylaxis for HIV infection integrated with municipal- and community-based sexual health services. JAMA Intern Med. 2016:176(1):75-84.

6. Scott HM, Klausner JD. Sexually transmitted infections and pre-exposure prophylaxis: challenges and opportunities among men who have sex with men in the US. Aids Res Ther. 2016;13(1):1-5.

7. Kretzschmar M, van Duynhoven YT, Severijnen AJ. Modeling prevention strategies for gonorrhea and chlamydia using stochastic network simulations. Am J Epidemiol. 1996;144(3):306-17.

8. Garnett GP. An introduction to mathematical models in sexually transmitted disease epidemiology. Sex Transm Infect. 2002;78(1):7-12.

9. Templeton DJ, Jin F, Imrie J, Prestage GP, Donovan B, Cunningham PH, Kaldor JM, Kippax S, Grulich AE. Prevalence, incidence and risk factors for pharyngeal chlamydia in the community based Health in Men (HIM) cohort of homosexual men in Sydney, Australia. Sex Transm Infect. 2008;84(5):361-3. 
10. Chow EP, Fairley CK. The role of saliva in gonorrhoea and chlamydia transmission to extragenital sites among men who have sex with men: new insights into transmission. J Int AIDS Soc. 2019;22(Suppl 6(Suppl Suppl 6)): e25354.

11. Cornelisse VJ, Fairley CK, Read TRH, Lee D, Walker S, Hocking JS, Chen MY, Bradshaw CS, Chow EPF. Associations between anorectal chlamydia and oroanal sex or saliva use as a lubricant for anal sex: a cross-sectional survey. Sex Transm Dis. 2018;45(8):506-10.

12. Althaus CL, Turner KME, Schmid BV, Heijne JCM, Kretzschmar M, Low N. Transmission of Chlamydia trachomatis through sexual partnerships: a comparison between three individual-based models and empirical data. J R Soc Interface. 2012;9(66):136-46.

13. Althaus $\mathrm{CL}$, Heijne JCM, Roellin A, Low N. Transmission dynamics of Chlamydia trachomatis affect the impact of screening programmes. Epidemics. 2010;2(3):123-31.

14. Vickerman P, Ndowa F, O'Farrell N, Steen R, Alary M, Delany-Moretlwe S. Using mathematical modelling to estimate the impact of periodic presumptive treatment on the transmission of sexually transmitted infections and HIV among female sex workers. Sex Transm Infect. 2010:86(3): $163-8$.

15. Regan DG, Wilson DP, Hocking JS. Coverage is the key for effective screening of Chlamydia trachomatis in Australia. J Infect Dis. 2008;198(3): 349-58.

16. Weiss KM, Jones JS, Katz DA, Gift TL, Bernstein K, Workowski K, Rosenberg ES, Jenness SM. Epidemiological impact of expedited partner therapy for men who have sex with men: a modeling study. Sex Transm Dis. 2019; 46(11):697-705.

17. Rekart ML, Ndifon W, Brunham RC, Dushoff J, Park SW, Rawat S, Cameron CE. A double-edged sword: does highly active antiretroviral therapy contribute to syphilis incidence by impairing immunity to Treponema pallidum? Sex Transm Infect. 2017;93(5):374-8.

18. Jenness SM, Weiss KM, Goodreau SM, Gift T, Chesson H, Hoover KW, Smith DK, Liu AY, Sullivan PS, Rosenberg ES. Incidence of gonorrhea and chlamydia following human immunodeficiency virus preexposure prophylaxis among men who have sex with men: a modeling study. Clin Infect Dis. 2017;65(5):712-8.

19. Xiridou M, Vriend HJ, Lugner AK, Wallinga J, Fennema JS, Prins JM, Geerlings SE, Rijnders BJ, Prins M, de Vries $\mathrm{HJ}$, et al. Modelling the impact of chlamydia screening on the transmission of HIV among men who have sex with men. BMC Infect Dis. 2013;13:436.

20. Tuli K, Kerndt PR. Preventing sexually transmitted infections among incarcerated men who have sex with men: a cost-effectiveness analysis. Sex Transm Dis. 2009;36(2 Suppl):S41-8.

21. van Liere G, Dukers-Muijrers N, Kuizenga-Wessel S, Götz HM, Hoebe C: What Is the Optimal Testing Strategy for Oropharyngeal Neisseria gonorrhoeae in Men Who Have Sex With Men? Comparing Selective Testing Versus Routine Universal Testing From Dutch Sexually Transmitted Infection Clinic Data (2008-2017). Clin Infect Dis. 2020;71(4):944-51.

22. Hiransuthikul A, Sungsing T, Jantarapakde J, Trachunthong D, Mills S, Vannakit R, Phanuphak P, Phanuphak N. Correlations of chlamydia and gonorrhoea among pharyngeal, rectal and urethral sites among Thai men who have sex with men: multicentre community-led test and treat cohort in Thailand. BMJ Open. 2019;9(6):e028162.

23. Tongtoyai J, Todd CS, Chonwattana W, Pattanasin S, Chaikummao S, Varangrat A, Lokpichart S, Holtz TH, van Griensven F, Curlin ME. Prevalence and correlates of Chlamydia trachomatis and Neisseria gonorrhoeae by anatomic site among urban Thai men who have sex with men. Sex Transm Dis. 2015;42(8):440-9.

24. Hinkan S, Chuerduangphui J, Ekalaksananan T, Budkaew J, Proyrungroj K, Pimson C, Chumworathayi B, Hanond T, Pientong C. Anatomical site distribution and genotypes of Chlamydia trachomatis infecting asymptomatic men who have sex with men in Northeast Thailand. Int J STD AIDS. 2018;29(9):842-50.

25. Rosenberger JG, Reece M, Schick V, Herbenick D, Novak DS, Van Der Pol B, Fortenberry JD. Sexual behaviors and situational characteristics of most recent male-partnered sexual event among gay and bisexually identified men in the United States. J Sex Med. 2011;8(11):3040-50.

26. de Vries R, van Bergen JE, de Jong-van den Berg LT, Postma MJ. Systematic screening for Chlamydia trachomatis: estimating cost-effectiveness using dynamic modeling and Dutch data. Value Health. 2006;9(1):1-11.
27. Zhang L, Regan DG, Chow EPF, Gambhir M, Cornelisse V, Grulich A, Ong J, Lewis DA, Hocking J, Fairley CK. Neisseria gonorrhoeae transmission among men who have sex with men: an anatomical site-specific mathematical model evaluating the potential preventive impact of mouthwash. Sex Transm Dis. 2017:44(10):586-92.

28. Spicknall $\mathrm{H}$, Mayer $\mathrm{KH}$, Aral SO, Romero-Severson EO. Assessing uncertainty in an anatomical site-specific gonorrhea transmission model of men who have sex with men. Sex Transm Dis. 2019;46(5):321-8.

29. Chow EP, Camilleri S, Ward C, Huffam S, Chen MY, Bradshaw CS, Fairley CK. Duration of gonorrhoea and chlamydia infection at the pharynx and rectum among men who have sex with men: a systematic review. Sex Health. 2016; 13(3):199-204.

30. Andersen B, Gundgaard J, Kretzschmar M, Olsen J, Welte R, Oster-Gaard L. Prediction of costs, effectiveness, and disease control of a population-based program using home sampling for diagnosis of urogenital Chlamydia trachomatis infections. Sex Transm Dis. 2006;33(7):407-15.

31. Jin F, Prestage GP, Mao L, Kippax SC, Pell CM, Donovan B, Cunningham PH, Templeton DJ, Kaldor JM, Grulich AE. Incidence and risk factors for urethral and anal gonorrhoea and chlamydia in a cohort of HIV-negative homosexual men: the Health in Men Study. Sex Transm Infect. 2007;83(2): 113-9.

32. Barbee LA, Khosropour CM, Dombrowski JC, Manhart LE, Golden MR. An estimate of the proportion of symptomatic gonococcal, chlamydial and non-gonococcal non-chlamydial urethritis attributable to oral sex among men who have sex with men: a case-control study. Sex Transm Infect. 2016; 92(2):155-60.

33. Rebe K, Lewis D, Myer L, de Swardt G, Struthers $H$, Kamkuemah M, Mclntyre J. A cross sectional analysis of gonococcal and chlamydial infections among men-who-have-sex-with-men in Cape Town, South Africa. PLoS One. 2015; 10(9):e0138315.

34. Fairley CK, Cornelisse VJ, Hocking JS, Chow EPF: Models of gonorrhoea transmission from the mouth and saliva. Lancet Infect Dis. 2019;19(10): e360-66.

35. Chow EPF, Cornelisse VJ, Read TRH, Lee D, Walker S, Hocking JS, Chen MY, Bradshaw CS, Fairley CK. Saliva use as a lubricant for anal sex is a risk factor for rectal gonorrhoea among men who have sex with men, a new public health message: a cross-sectional survey. Sex Transm Infect. 2016;92(7):532-6.

36. Newcombe RG. Two-sided confidence intervals for the single proportion: comparison of seven methods. Stat Med. 1998;17(8):857-72.

37. Hu FB. Diet and exercise for new-onset type 2 diabetes? Lancet. 2011; 378(9786):101-2

38. Wilson EB. Probable inference, the law of succession, and statistical inference. J Am Stat Assoc. 1927;22(158):209-12

39. Chow EP, Tabrizi SN, Phillips S, Lee D, Bradshaw CS, Chen MY, Fairley CK. Neisseria gonorrhoeae bacterial DNA load in the pharynges and saliva of men who have sex with men. J Clin Microbiol. 2016;54(10):2485-90.

40. Read TRH, Murray GL, Danielewski JA, Fairley CK, Doyle M, Worthington K, Su J, Mokany E, Tan LT, Lee D, et al. Symptoms, sites, and significance of mycoplasma genitalium in men who have sex with men. Emerg Infect Dis. 2019;25(4):719-27

41. Footman A, Dionne-Odom J, Aaron KJ, Raper JL, Van Der Pol B: Performance of 4 Molecular Assays for Detection of Chlamydia and Gonorrhea in a Sample of Human Immunodeficiency Virus-Positive Men Who Have Sex With Men. Sex Transm Dis 2020, 47(3):158-161.

42. BVD P. Extragenital CT/GC in MSM. In: The 20th International Union against Sexually Transmitted Infections- Asia Pacific Conference (IUSTI-AP): 2019 Shanghai; 2019.

43. Paige CC, Saunders MA. LSQR: an algorithm for sparse linear equations and sparse least squares. ACM Trans Math Softw. 1982;8(1):43-71.

44. Onwubu SC, Mdluli PS, Singh S, Collins OC. The application of the logistic equation model to predict the remineralization characteristics of desensitizing paste. Int J Dent. 2019;2019:7528154

45. Phillips TR, Fairley CK, Maddaford K, Danielewski J, Hocking JS, Lee D, Williamson DA, Murray G, Kong F, De Petra $V$ et al: Bacterial Load of Chlamydia trachomatis in the Posterior Oropharynx, Tonsillar Fossae, and Saliva among Men Who Have Sex with Men with Untreated Oropharyngeal Chlamydia. J Clin Microbiol. 2019;58(1):e01375-01319.

46. Dukers-Muiirers N, Wolffs PFG, de Vries HJC, Götz HM, Janssen K, Hoebe C. Viable bacterial load is key to azithromycin treatment failure in rectally Chlamydia trachomatis infected women (FemCure). J Infect Dis. 2019;220(8): 1389-90. 
47. Wyrick PB. Chlamydia trachomatis persistence in vitro: an overview. J Infect Dis. 2010;201(Supplement_2):S88-95.

48. Lewis D, Newton DC, Guy RJ, Ali H, Chen MY, Fairley CK, Hocking JS. The prevalence of Chlamydia trachomatis infection in Australia: a systematic review and meta-analysis. BMC Infect Dis. 2012;12:113.

49. Ong JJ, Chow EPF, De Petra V, Williamson D, Pelatosis I, Howden B, Zhang L, Chen MY, Bradshaw CS, Hocking J, et al. Should asymptomatic men who have sex with men be screened for oropharyngeal chlamydia? Clinical outcomes from a cross-sectional study. Sex Transm Dis. 2018;45(2):103-6.

50. Dolat L, Valdivia RH. A renewed tool kit to explore Chlamydia pathogenesis: from molecular genetics to new infection models. F1000Res. 2019:8:F1000 Faculty Rev-1935.

51. Peters RP, Verweij SP, Nijsten N, Ouburg S, Mutsaers J, Jansen CL, van Leeuwen AP, Morre SA. Evaluation of sexual history-based screening of anatomic sites for Chlamydia trachomatis and Neisseria gonorrhoeae infection in men having sex with men in routine practice. BMC Infect Dis. 2011;11:203.

52. Ribeiro S, de Sousa D, Medina D, Castro R, Lopes A, Rocha M. Prevalence of gonorrhea and chlamydia in a community clinic for men who have sex with men in Lisbon, Portugal. Int J Std Aids. 2019;30(10):951-9.

\section{Publisher's Note}

Springer Nature remains neutral with regard to jurisdictional claims in published maps and institutional affiliations.

- fast, convenient online submission

- thorough peer review by experienced researchers in your field

- rapid publication on acceptance

- support for research data, including large and complex data types

- gold Open Access which fosters wider collaboration and increased citations

- maximum visibility for your research: over $100 \mathrm{M}$ website views per year

At $\mathrm{BMC}$, research is always in progress. 